\title{
A genetic dichotomy model for the inheritance of Alzheimer's disease and common age-related disorders
}

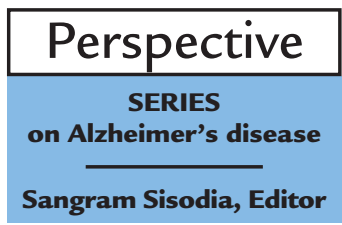

\author{
Rudolph E. Tanzi \\ Genetics and Aging Research Unit, Massachusetts General Hospital, \\ Harvard Medical School, Building 149, 13th Street, Charlestown, Massachusetts 02129, USA. \\ Phone: (617) 726-6845; Fax: (617) 726-5677; E-mail: tanzi@helix.mgh.harvard.edu.
}

Alzheimer's disease (AD), already one of the most serious health problems in the U.S., will carry an ever burgeoning impact as the proportion of elderly continues to increase. Clinically, $\mathrm{AD}$ is a progressive neurodegenerative disorder characterized by global cognitive decline. Neuropathologically, the brains of AD patients contain abundant amounts of neurofibrillary tangles (NFT) and $\beta$-amyloid in the form of senile plaques (SP) and blood vessel deposits. While the etiological events that lead to AD have not been clearly resolved, genetic factors clearly play a major role; there is an emerging consensus that $\mathrm{AD}$ is a complex and genetically heterogeneous disorder that is best explained by an age-dependent dichotomous model. On one hand, early-onset $(<60) \mathrm{AD}$ is caused by defects in any of three different genes: presenilin 1 (PSEN1) on chromosome 14 (1), presenilin 2 (PSEN2) on chromosome 1 (2), and the amyloid $\beta$ protein precursor $(A P P)$ on chromosome 21 (3). On the other hand, late-onset $\mathrm{AD}$ is associated with genetic polymorphisms that appear to operate as risk factors and/or genetic modifiers.

Genetic linkage analyses have led to the identification of two such genetic risk factors: the apolipoprotein E gene (APOE) on chromosome 19 (4) and alpha2macroglobulin $(A 2 M)$ on chromosome $12(5)$. On the basis of these and other findings, I describe in this Perspective a genetic dichotomy model for the inheritance of AD. This model, which is also useful for defining the genetic basis of other common, age-related disorders (e.g. cardiovascular disease, cancer, and diabetes), is based on the simple concept that while rare, autosomal dominant (or recessive) forms of age-related diseases are caused by rare, often $100 \%$ penetrant mutations that confer severe biological impact, the late-onset, more common forms of these disorders occur with increasing frequency with advanced age and are associated with genetic risk factors in the form of common population polymorphisms (CPP) distributed throughout the human genome.

Together, the CPPs that predispose to common agerelated diseases like $\mathrm{AD}$ can eventually be used to develop a genotypic profiling protocol. Such a protocol would have to be performed in a confidential setting by licensed clinical laboratories with appropriate genetic and psychological counseling, as well as legal safeguards, in place. Moreover, assessing one's relative risk for $\mathrm{AD}$ and other age-related diseases would be warranted only if effective means were also available for preventing or treating the disease, preferably according to a pharmacogenomic (or nutrigenomic) strategy customized to one's specific genotypic profile

Early-onset $A D$ : A rare disorder caused by rare highly penetrant mutations. Early efforts to understand the role of genetics in AD in the early 1980s focused on rare multigenerational families with early-onset, autosomal dominant, fully penetrant forms of the disease. Eight different pathogenetic mutations have been identified in $A P P$, and all are missense mutations lying within or close to the domain encoding the $A \beta$ peptide, the major component of $\beta$-amyloid in $\mathrm{AD}$. The APP mutations account for less than $0.1 \%$ of $\mathrm{AD}$ cases (6) but carry severe biological consequences. The age of onset of $\mathrm{AD}$ reported for individuals harboring pathogenic mutations in the APP gene ranges from 39 to 67 , with some differences in mean age-at-onset for the different specific mutations. APP mutations are $100 \%$ penetrant in the vast majority of carriers.

A substantial portion ( $40 \%)$ of early-onset $\mathrm{AD}$ is associated with mutations in PSEN1 and PSEN2, primarily in PSEN1 (6; see also Thinakaran, in the next issue of the JCI). To date, 64 different $\mathrm{AD}$ mutations have been reported in PSEN1 in over 130 unrelated kindreds or singleton cases of $\mathrm{AD}$, while only four PSEN2 AD mutations have been reported (6). All presenilin mutations are missense mutations (single amino acid changes), with the exception of two, one of which is an insertion/deletion mutation (owing to the activation of a cryptic splice donor) in the N-terminus of PSEN1 and the other of which consists of a missense mutation resulting from the deletion of exon 10. With only 2 exceptions, all pathogenic missense mutations in the presenilins occur in residues that are conserved between the two proteins. All but eight mutations in PSEN1 occur within or immediately juxtaposed with either the eight predicted transmembrane domains or the hydrophobic stretch of amino acids between predicted transmembrane domains six and seven. This latter stretch is believed to enter and exit the membrane on the cytoplasmic face of the protein. The greatest concentration of mutations occurs in or around predicted transmembrane domains six and seven and the hydrophobic cluster sitting between these domains. Of the eight mutated residues that lie elsewhere in the pro- 
tein, four are located in the luminal loop between predicted transmembrane domains one and two, two are in the large cytoplasmic loop between predicted transmembrane domains six and seven, and two are in the carboxy-terminal cytoplasmic tail.

Three of the four mutations in PSEN2 also reside within predicted transmembrane domains. Thus, even though these mutations are distributed throughout the presenilins, their primary pathogenic effect appears to be elicited in the membrane-spanning or hydrophobic portions of these proteins, perhaps affecting their interactions with other hydrophobic helices (e.g. within intracellular membranes), where they primarily reside (7). Thus, one common consequence of the presenilin $\mathrm{AD}$ mutations may be to alter the conformation of these proteins, perhaps making them more prone to intracellular aggregation, a common feature of a variety of mutated proteins associated with neurodegenerative diseases. How misfolding of FAD-associated mutant presenilins and their increased susceptibility to aggregation might be related to increased production of $\mathrm{A} \beta 42$, a major pathogenic consequence of these mutations, remains unresolved. All but one of the PSEN1 mutations are $100 \%$ penetrant and are sufficient (but not necessary) to cause $\mathrm{AD}$. The mean age of onset of $\mathrm{AD}$ in PSEN1-linked AD families is approximately 45 years with a range of 28 to 60 years. In contrast, the mean age of onset in families for the N141I PSEN2 mutation (the Volga Germans) is 52 years, and individual ages of onset in these kindreds range from 40-85 years. The early-onset $\mathrm{AD}$ mutations are associated with disastrous phenotypic consequences that are apparent at a relatively early point in adult life. Such mutations therefore cause a profound biological impact and are virtually $100 \%$ penetrant, but they are exceedingly rare.

Late-onset $A D$ : A common age-related disorder associated with $C P P s$. In contrast with the relative simplicity of the genetics of early-onset $\mathrm{AD}$, which represents only a small fraction of the disorder, the genetics of the far more common late-onset form of the disease is considerably more complex. Even so, several lines of genetic epidemiological evidence from family, twin, and segregation studies indicate that genes play a major role in its etiology, and genetic studies have arguably represented the principal means of learning about this devastating disease. In addition to its complex genetics, several factors contribute to the difficulty of studying genes involved in late-onset AD. First, the base rate of the disorder is high and rises steeply with age. Thus, some clustering in families may be due to chance alone. Second, late-onset AD occurs very near the end of the life span, so that many individuals do not survive the age of risk. This has made it difficult to assess the mode of inheritance of late-onset $\mathrm{AD}$. Third, elderly patients are at greater risk of other causes of cognitive decline, making the risk of phenocopies somewhat higher in this age group.

To date, late-onset $\mathrm{AD}$ has been genetically characterized by increased risk owing to the inheritance of common DNA variants in the genome. Base-pair variations occur in the human genome on average once every thousand bases, culminating in roughly 3 million DNA sequence differences between any two random individuals. While many, if not most, of these variations are innocuous at present, a fair number of them appear to predispose to common age-related diseases such as cardiovascular disease, cancer, diabetes and $\mathrm{AD}$. As the human lifespan continues to increase, it is reasonable to expect that some of these common DNA variants will become medically relevant in the future. Some of these variations, referred to here as CPPs, are relatively common in the general population, with an average prevalence of $10 \%$ or more. The basic premise proposed in this perspective is that common age-related disorders such as $\mathrm{AD}$, cancer, cardiovascular disease, and diabetes, are genetically associated with varying degrees of risk owing to the inheritance of various CPPs.

The APOE gene and risk for late-onset $A D$. The $A P O E$ gene contains the first CPP to be genetically associated with increased risk for late-onset $\mathrm{AD}$. Of the three major $A P O E$ gene alleles, the $\varepsilon 4$ allele is associated with $\mathrm{AD}(4)$. Employing case-control (population-based) association studies, the APOE- $\varepsilon 4$ allele was found to be over-represented in $\mathrm{AD}$ cases, thereby implicating it as a risk factor (4). This association was subsequently confirmed in numerous other case-control studies. In one study of 310 families collected through the auspices of the NIMH Genetics Initiative (8), APOE- $\varepsilon 4$ was also confirmed as a risk factor for $\mathrm{AD}$ using genetic linkage analysis and conditional logistic regression. This study (8) also showed that the effect of $A P O E-\varepsilon 4$ is age-dependent, with a peak effect in the seventh decade, a finding that was later confirmed in a large meta-analysis.

While APOE- $\varepsilon 4$ has been confirmed as a strong risk factor for $\mathrm{AD}$, it is clearly not necessary for the development of AD. Some have estimated that this allele accounts for $50 \%$ of the susceptibility to AD (4), but such estimates must be interpreted with caution. First, APOE- $\varepsilon 4$ is present in $20-30 \%$ of the general population, so its presence in an $\mathrm{AD}$ subject does not necessarily mean it is responsible for the disease. Second, even homozygosity for APOE- $\varepsilon 4$ is not sufficient to cause the disease. In fact, Blacker et al. (8) identified 15 individuals with two copies of this allele who were as much as 10 years older than their two affected siblings but were cognitively normal. Third, the APOE- $\varepsilon 4$ allele appears to act primarily as a modifier of age at onset of AD (9). Thus, it is more reasonable to conclude at this juncture that this allele is just one of probably a large set of CPPs contributing to age-related risk for lateonset $\mathrm{AD}$. In considering other $\mathrm{CPP}$ that contribute to risk for $\mathrm{AD}$, three other $A P O E \mathrm{CPPs}$ in addition to $A P O E-\varepsilon 4$ have been reported to be associated with $\mathrm{AD}$, including two in the promoter $(10,11)$ as well as a missense mutation (P28L; 12). It is not clear as to which of these $\mathrm{AD}$-associated $A P O E$ CPPs are actually pathogenic for predisposition to $\mathrm{AD}$, as opposed to being in linkage disequilibrium with other, pathogenic CPPs. This quandary illustrates the critical need for hard bio- 
logical data demonstrating the mechanism by which any or all four of the currently identified AD-associated $A P O E$ CPPs promote AD neuropathogenesis.

Toward the development of a genotypic profile for $A D$. Following the identification of $A P O E$ as a risk factor for $\mathrm{AD}$, a large number of genes and their respective CPPs were similarly tested for association with $\mathrm{AD}$ using the case-control design. With the exception of APOE and $A 2 M$, all the late-onset $\mathrm{AD}$ candidate genes were initially tested primarily on the basis of biological hypotheses regarding their potential involvement in the disease process. In contrast, the positive results of genetic linkage studies prompted the testing of APOE (chromosome 19 linkage) and $A 2 M$ (chromosome 12 linkage). If confirmed as true genetic risk factors for $\mathrm{AD}$, the CPPs in many of these genes could be considered for inclusion in the emerging genotypic profile for late-onset $A D$. To date, $C P P s$ associated with increased risk for $A D$ have been identified in the genes for $\alpha 1$-antichymotrypsin $(A C T)$, the very low density lipoprotein receptor $(V L D L R)$, the LDL-receptor related protein $(L R P)$, PSEN1 (an intronic polymorphism in intron 9), alpha2macroglobulin $(A 2 M)$, bleomycin hydrolase $(B H)$, the estrogen receptor $\alpha(\mathrm{ER}-\alpha)$, neurotrophin-3 (NT3), myeloperoxidase $(M P O)$ the serotonin receptor (5HTR), the non-amyloid component of plaques $(N A C P)$, dihydrolipoyl succinyltransferase (DLST), N-acetyltransferase $(N A T)$, angiotensin converting enzyme $(D C P)$, FE65 (APPBP1), cathepsin D (CATD), HLA (the HLA-A2 allele), transferrin (TF), butyrylcholinesterase (BCHE-K variant), and lipoprotein lipase ( $L P L)$. Furthermore, decreased risk for $\mathrm{AD}$ was associated with a CPP in interleukin 6 (IL6). In addition, certain mitochondrial genome alterations have been associated with increased risk for AD. Finally, CPPs in the 5-HT2A and 5-HT2C receptor genes have been associated with psychotic symptoms in $\mathrm{AD}$ patients. Thus, in addition to the CPPs in APOE, CPPs in roughly two dozen other genes have been associated with late-onset $\mathrm{AD}$ and have armed us with useful preliminary data necessary to begin further research and development of a genotypic profile for pre-symptomatic diagnosis of AD.

With the exception of $A 2 M$, associations between CPPs in the above genes and altered risk or specific clinical symptoms of $\mathrm{AD}$ were derived from case-control studies. Importantly, none of the CPPs in any of these genes has been as robustly replicated or universally confirmed for association with $\mathrm{AD}$ as APOE-E4. This does not necessarily mean that all these other gene associations with $\mathrm{AD}$ represent false positives but rather reflects the limited utility and reliability of the population-based association (case-control) method in which allele frequencies are simply compared between sets of cases and controls to test for statistically significant differences in representation of certain alleles. Casecontrol studies are particularly prone to type II errors. One source of error in this form of association analysis is population admixture. Skewed distributions of ethnic etiologies and/or the presence of distinct predis- posing etiologies within and between the case and control sample sets can lead to inconsistent results from one case-control study to the next. The late onset of AD introduces other potential pitfalls: the age at which cases and controls are assessed for association is critical, since certain genetic risk factors (e.g. A2M) may increase susceptibility to $\mathrm{AD}$ only at very old age.

For these reasons, it is not unexpected for an initial report of an association between a CPP and a heightened risk of $\mathrm{AD}$ to be confirmed in 2-3 follow-up studies but to be refuted in 5 or more such studies. Such inconsistent findings should not be seen as prima facie evidence that the original report of association was false. The likelihood that three different association studies would somehow lead to false positive results is relatively low. More probably, the failure of some studies to replicate the association can be explained by a combination of several factors. First, the CPP itself may be pathogenically associated with $\mathrm{AD}$ but only in a subgroup of carriers; other risk factors, genetic or environmental, may be simultaneously required for expression of the disease phenotype, and these latter influences may operate inconsistently in different populations. Second, the CPP being tested may not represent a disease allele of a gene related to $\mathrm{AD}$, but may instead be in linkage disequilibrium with such a CPP residing in either the same gene or in one nearby. This linkage disequilibrium relates to the history of a population and would be expected to vary between ethnic groups. A case in point is the reported association of $\mathrm{AD}$ with FE65 and Cathepsin D, genes that reside side by side on chromosome 11. Most probably, only one of these genes actually contains a CPP or mutation that is pathogenically associated with $\mathrm{AD}$, while the other is only in linkage disequilibrium. In this example, both genes are biologically sound candidates as risk factors for AD. FE65 affects the processing of APP, and cathepsin D can cleave APP at the A $\beta$ domain in cultured cells. More genetic studies are necessary to determine the correct $\mathrm{AD}$-associated gene on chromosome. Thus, even in the face of multiple refutations, it is reasonable to follow up confirmed associations by testing additional samples and including the examination of neighboring genes. The ultimate confirmation of any candidate gene requires pathobiological data directly implicating the associated allele in the disease process.

Considerations in the search for novel associations with $A D$ : The case of $A 2 M$. The optimal strategy for a genomic screen for a complex disease like $\mathrm{AD}$ is one that achieves good power with reasonable efficiency. For the analysis of preliminary genome screen results, a combination of full likelihood lod score analysis (referred to as "parametric") and partial likelihood analysis with little explicit modeling of the mode of inheritance (referred to as "non-parametric") should be employed. With regard to the former, it can be argued that since there is sufficient information regarding the genetics of early-onset $\mathrm{AD}$ to support a dominant model, some, albeit limited, additional power could be extracted from the full likelihood 
model. In the latter case, a non-parametric approach (e.g. using the program GENEHUNTER, which does not specify the mode of inheritance), would have a greater chance of identifying genes of more modest effect, or whose actions are non-Mendelian, such as those likely to be involved in late-onset AD. Stratifications, based on the combination of these two primary modes on age or APOE status, can also increase the odds of identifying novel associations. However, careful testing for the effects of multiple comparisons would be necessary, using the Bonferroni correction.

Employing the 2-tiered parametric and non-parametric approach, a linkage signal for late onset $\mathrm{AD}$ was previously detected on the short arm of chromosome 12 in the NIMH AD family sample. One of the candidate genes residing in the linkage area was $A 2 M$. The alpha2-macroglobulin $\left(\alpha_{2} M\right)$ protein is a serum panprotease inhibitor that inhibits all four classes of proteases by steric trapping (in which the protease remains active). Over the past several years, an impressive array of a priori biological evidence supporting the candidacy of $A 2 M$ as a potential genetic risk factor for $\mathrm{AD}$ has amassed. Briefly, $\alpha_{2} \mathrm{M}$ has been demonstrated to mediate the clearance and degradation of $\mathrm{A} \beta$ either directly (in complex with serine proteases) or by means of clearance through LRP. Genetic analysis of a CPP consisting of a pentanucleotide deletion in the $5^{\prime}$-splice site of exon 2 of the $A 2 M$ gene revealed that inheritance of the deletion allele (A2M-2) conferred increased risk for AD in the NIMH sample (5).

The reported association between $\mathrm{AD}$ and $\mathrm{A} 2 \mathrm{M}$ was unique in the sense that it was the first AD-related gene to be initially identified using "family-based" as opposed to "population-based" association analyses. Specifically, the sibship disequilibrium test (SDT; 13), which compares the inheritance of alleles of a given gene CPP among affected and unaffected siblings, was employed. The SDT does not require parental data and is similar in form to the sibship-transmission disequilibrium test (S-TDT; 14). There are two major advantages to the family-based association approach. First, when a CPP (or DNA marker) and a disease locus are tightly linked, it is powerful enough to detect an association, even when traditional genetic linkage analyses (parametric or non-parametric) lack the power necessary to detect linkage. The latter condition is often the case with CPPs, which are usually not particularly informative. Second, these analyses usually protect against spurious associations that can result in case-control studies caused by population stratification. This is because the genetic background of the affected and unaffected individuals being compared is relatively normalized (as they are either siblings or first-degree relatives).

In the original family-based association analysis of $A 2 M$ and $\mathrm{AD}$ using the SDT, a highly significant association was observed between $A 2 M-2$ and $\mathrm{AD}$, similar to that observed for the $A P O E-\varepsilon 4$ allele in the same set of NIMH families (5). Subsequent to the publication of the association of $A 2 M$ and $\mathrm{AD}$, Rudrasingham and colleagues (15) corroborated the association using the NIA family sample and employing family-based association analysis. In addition, three different familybased association analyses of overlapping subsets of the NIMH sample yielded statistically significant associations between $A 2 M$ and $\mathrm{AD}(15-18)$. In addition to the replication of family-based association of $A 2 M-2$ and $\mathrm{AD}$, multiple linkage studies have also shown linkage peaks in the $A 2 M$ region of chromosome 12 (see, for example, ref. 19).

In a smaller set of families and in a number of casecontrol analyses, no significant association was observed between $A 2 M$ and AD (15-17). These contradictory findings highlight some of the differences between family-based versus case-control association studies. The family-based association design estimates the magnitude of the effect in the context of other shared familial factors, whether they be genetic or, to a more limited extent, environmental. Thus, family-based association is an effective tool for identifying genetic modifiers for disease that operate in the context of other shared genetic and, to a lesser extent, environmental factors shared by family members. Such genetic modifiers could easily be missed in population-based association studies, which analyze a random assortment of individuals with diverse genetic backgrounds. Nonetheless, it is worth noting that in two recent case-control studies, another CPP, at amino acid position 1000 (V/I) of $A 2 M$, was found to be associated with $\mathrm{AD}$ as well as increased $\beta$-amyloid deposition $(20,21)$. A2M thus becomes the fifth gene (in addition to APP, PSEN1, PSEN2, and $A P O E)$ that contains DNA sequence variations associated with significantly increased accumulation of $\beta$-amyloid in AD brains of carriers versus non-carriers. These data provide further support to the notion that increased accumulation of $\beta$-amyloid in the brains of $\mathrm{AD}$ patients who carry new mutations and CPPs genetically associated with $\mathrm{AD}$ can be used to authenticate a proposed genetic association.

The study by Myllykangas et al. (21) is also interesting with regard to the light it sheds on considerations that should be taken into account when testing the genetic association between $A 2 M$ and $\mathrm{AD}$, considerations that may be generally applicable to association studies of AD and other age-related disorders. In that study, a statistically significant association between $A 2 M$ and AD was observed in the neuropathologically confirmed $\mathrm{AD}$ cases, but not in those that were assessed solely by clinical examination. Additionally, the subjects in this positive study were very old (> 85) and mainly female (80\%). These findings thus serve to underscore the importance of considering the effects of age, gender, and confirmation of disease status on the outcome of genetic studies of $\mathrm{AD}$ and most probably other age-related disorders.

\section{Conclusions}

Genetic studies of AD have already provided valuable insights into this complex disorder and are beginning to 
provide clues regarding molecular mechanisms of the disease. Mutations in APP, PSEN1, and PSEN2 have severe biological consequences and confer early-onset $\mathrm{AD}$ in an autosomal dominant fashion with virtually $100 \%$ certainty. In contrast with the early-onset mutations, CPPs in a host of genes, most notably $A P O E$ and $A 2 M$, have been associated with late-onset $\mathrm{AD}$. These appear to act as risk factors and/or genetic modifiers of age-at-onset $(A P O E)$ or of particular phenotypic features, e.g., amyloid deposition ( $A P O E$ and $A 2 M)$. Unlike the highly penetrant, autosomal dominant mutations in APP, PSEN1, and PSEN2, the CPPs in APOE and A2M only increase risk for $\mathrm{AD}$ but do not guarantee onset, and the biological impact of these CPPs pales in comparison with the other mutations. Furthermore, these CPPs probably require the presence of other genetic and/or environmental risk factors for expression of the disease phenotype.

The genetic dichotomy model of AD presented here can be generalized to other disorders that are agedependent but that can also strike early in life (e.g., cardiovascular disease, cancer, diabetes, Parkinson's disease). While rare forms of age-related diseases can be caused by rare, highly penetrant mutations with severe biological impact, much more common forms of the disease that occur with increasing age are associated with genetic risk factors in the form of CPPs distributed throughout the human genome. On the basis of this dichotomy, the genomic alterations genetically linked to or associated with AD can be broadly classified according to three basic criteria: prevalence (or incidence), penetrance, and pathobiological impact (as reflected by age of onset). This classification system supports a genetic dichotomy of the inheritance of AD in which autosomal dominant mutations in APP and the presenilins would be classified as extremely rare (low prevalence), highly penetrant, and of very high pathobiological impact (early age of onset). In contrast, the CPPs associated with the APOE and $A 2 M$ genes are of relatively high prevalence (> $10 \%$ ), are weakly penetrant, and carry low biological impact, as judged by the relatively late onset of symptoms.

Ultimately, the identification of disease-associated CPPs for AD and other age-related disorders will permit the development of genotypic profiling protocols that can be reliably employed to predict late-life onset of age-related disorders This development in our prognostic ability will have to be applied with great care. Analysis will have to be provided in a confidential clinical setting that includes genetic and psychological counseling and appropriate legal safeguards regarding the genotypic information. Testing for specific genotypic profiles will only be warranted if it can be used to tailor pharmaceutical or nutriceutical strategies to prevent or treat these late-onset disorders. Identifying genes for late-onset $\mathrm{AD}$ will also require the continual development and employment of powerful analytical approaches. For example, the false positive results which often result from population-based (case-control) studies can be dramatically minimized by employ- ing more discordant sibships and family-based association analyses in the future, e.g., utilizing SDT (13) or S-TDT (14) analyses.

With the identification of additional genes involved in $\mathrm{AD}$, investigators and clinicians involved in $\mathrm{AD}$ research will be afforded an increasingly greater opportunity to piece together the puzzle of AD pathophysiology. More significantly, they will have the chance to develop more effective strategies for treatment and prevention of $\mathrm{AD}$ based specifically on an individual's inherited set of ADassociated CPPs. Ultimately, customized genotype-guided treatments will be our very best lines of defense and offense against this formidable disease.

\section{Acknowledgments}

Given the limitation in the number of references that can be included in this Perspective, it was not possible to provide citations for all presenilin FAD mutations or for the list of genes containing CPPs that have been associated with increased risk for AD.

1. Sherrington, R., et al. 1995. Cloning of a novel gene bearing missense mutations in early familial Alzheimer disease. Nature. 375:754-760.

2. Levy-Lehad, E., and Wasco, W., et al. 1995. Candidate gene for the chromosome 1 familial Alzheimer's disease locus. Science. 269:973-977.

3. Goate, A.M., et al. 1991. Segregation of a missense mutation in the amyloid precursor protein gene with familial Alzheimer's disease. Nature. 349:704-706.

4. Saunders, A.M., et al. 1993. Association of Apolipoprotein E allele Epsilon 4 with late-onset Alzheimer's disease. Neurology. 43:1467-1472.

5. Blacker, D., et al. 1998. Alpha2-macroglobulin is genetically associated with Alzheimer's disease. Nat. Genet. 19:357-360.

6. Tanzi, R.E., et al. 1996. The gene defects responsible for Familial Alzheimer's Disease. Neurobiol. Dis. 3:159-168.

7. Kovacs, D.M., et al. 1996. Alzheimer associated presenilins 1 and 2: neuronal expression in brain and localization to intracellular membranes in mammalian cells. Nat. Med. 2:224-229.

8. Blacker, D., et al. 1997. APOE-4 and age-at-onset of Alzheimer's disease: the NIMH Genetics Initiative. Neurology. 48:139-147.

9. Meyer, M.R., et al. 1998. APOE genotype predicts when - not whether one is predisposed to develop Alzheimer disease. Nat. Genet. 19:321-322.

10. Bullido, M.J., et al. 1998. A polymorphism in the regulatory region of APOE associated with risk for Alzheimer's dementia. Nat. Genet. 18:69-71

11. Lambert, J.C., et al. 1998. A new polymorphism in the APOE promoter associated with risk of developing Alzheimer's disease. Hum. Mol. Genet. 7:533-540.

12. Kamboh, M.I., et al. 1999. A novel mutation in the apolipoprotein E gene (APOE*4 Pittsburgh) is associated with the risk of late-onset Alzheimer's disease. Neurosci. Lett. 26:129-132.

13. Horvath, S., and Laird, N.M. 1998. A discordant sibship test for disequilibrium and linkage: no need for parental data. Am. J. Hum. Genet. 63:1886-1897.

14. Spielman, R.S., Ewens, W.J. 1998. A sibship test for linkage in the presence of association: the Sib Transmission/Disequilibrium Test. Am.J. Hum. Genet. 62:450-458.

15. Rudrasingham, V., et al. 1999. Alpha-2 macroglobulin gene and Alzheimer's disease. Nat. Genet. 22:17-19.

16. Dow, D.J., et al. 1999. Alpha-2 macroglobulin and Alzheimer disease risk in the UK. Nat. Genet. 22:16-17.

17. Rogaeva, E., et al. 1999. A comprehensive examination of an alpha-2macroglobulin insertion-deletion polymorphism in Alzheimer disease. Nat. Genet. 22:19-21.

18. Blacker, D., Crystal, A.S., Wilcox, M.A., Laird, N.M., and Tanzi, R.E. 1999. Alpha-2-macroglobulin gene and Alzheimer's disease. Nat. Genet. 22:16-17.

19. Scott, W.K., et al. 1999. Further evidence linking late-onset Alzheimer's disease with chromosome 12. JAMA. 281:513-514.

20. Liao, A., et al. 1998. Genetic association of an alpha-2-macroglobulin polymorphism and Alzheimer's disease. Hum. Mol. Genet. 7:195-196.

21. Myllykangas, L., et al. 1999. Genetic association of alpha2-macroglobulin with Alzheimer's disease in a Finnish elderly population. Ann. Neurol. 43:382-390. 